\title{
3 Researc Square

\section{The Impact of Vertical Off-Centring, Tube Voltage and Phantom Size on CT Numbers: An Experimental Study}

Yazan Al-Hayek ( $\nabla$ yazan.m.alhayek@gmail.com )

Charles Sturt University https://orcid.org/0000-0003-3985-1796

Kelly Spuur

Charles Sturt University

Rob Davidson

University of Canberra

Christopher Hayre

Charles Sturt University

Xiaoming Zheng

Charles Sturt University

\section{Research Article}

Keywords: CT number, off-centring, phantom size, tube voltage, and uniformity.

Posted Date: July 7th, 2021

DOI: https://doi.org/10.21203/rs.3.rs-658343/v1

License: (9) This work is licensed under a Creative Commons Attribution 4.0 International License.

Read Full License 


\section{Abstract}

Objectives: This pilot experimental study explores the effect of vertical off-centring on CT numbers in combination with various tube voltages and phantom sizes.

Methods: A CIRS Model 062 Electron Density and Combined Head and Body phantom underwent imaging using a Siemens Emotion 16-slice CT and GEMINI GXL scanners. Uniformity was evaluated as a function of vertical off-centring $(20,40,60$, and $80 \mathrm{~mm}$ above the gantry iso-centre) using different phantom sizes and tube voltage for ROI positions across the $X$ and $Y$ axis of each phantom. CT number change was assessed by comparing the measured values between anterior (upper) versus posterior (lower) ROls.

Results: The results showed that vertical off-centring and phantom size could account for $92 \%$ of the recorded variance and resultant CT number change. The uniformity test recorded a maximum change of 27.2 and $14 \mathrm{HU}$ for peripheral ROls at $80 \mathrm{~mm}$ phantom shift above the gantry iso-centre using the GEMINI GXL and Siemens scanners, respectively. The absolute CT number differences between anterior and posterior ROls were 13.7 for the $30 \mathrm{~cm}$ phantom and 4.8 for the $20 \mathrm{~cm}$ phantom at $80 \mathrm{~mm}$ vertical offcentring. The most remarkable differences were observed at lower tube voltages.

Conclusions: It is essential to highlight the significance of optimal patient centring for CT examinations and the consequences of CT numbers variation on clinical decision making. Phantom off-centring and ROI location have been demonstrated to affect CT number uniformity in this pilot experimental study. This was more evident at peripheral phantom areas, lower tube voltages and larger phantom size.

\section{Introduction}

Computed tomography (CT) has long been considered an important imaging modality providing characterisation of the composition and anatomical location of soft-tissue lesions [1]. The reliability of Quantitative CT (QCT) to assess diseases is primarily dependent upon the accuracy of the CT number (or the Hounsfield Unit (HU)) of the tissue scanned [2-4]. In CT, the Hounsfield scale is used to represent all body tissues according to a linear density scale, with water arbitrarily assigned a value of zero $\mathrm{HU}$, and all other values computed according to the following formula [5]:

$$
H U=\frac{\mu_{\text {tissue }}-\mu_{\text {water }}}{\mu_{\text {water }}} \times 1000
$$

However, it is widely accepted that CT numbers are influenced by several factors, including scanner design and calibration, reconstruction algorithms, beam hardening artifacts, body size, object orientation, the tube voltage, and object off-centring [6-10]. These factors are known to cause CT number variability, which can significantly affect the reliability of the resultant data [11]. Importantly, variability in the CT number can affect the sensitivity and specificity of lesion detection and characterisation which are based on the CT number threshold [12], such as adrenal and renal masses [13-15]. Furthermore, the calculated 
radiation dose for therapy may also be affected by CT number variability because dose calculation depends primarily on the CT number in obtaining mass density of lesion under treatment [16].

Body size is also considered a factor that contributes to the inaccuracy of the CT number as a result of a beam hardening effect [17] and scanner calibration [18]. Consequently, various beam hardening correction schemes are incorporated into modern clinical scanners. However, these corrections are calibrated with one specific body size and tube voltage only [18].

In CT, optimal patient positioning requires the patient to be oriented at the iso-centre of the scanner gantry. However, it is well known that off-centring in the clinical setting commonly occurs, most notably in the vertical direction, below the gantry iso-centre $[19,20]$. Correct centring is important as bowtie filters are used to adjust the distribution of the beam based on scanned body attenuation. Even beam distribution is typically achieved by restricting the beam intensity at the periphery, thus allowing a higher central beam intensity [21]. To maximum the bowtie filter effect, patient centring needs to be precise.

Several studies have shown that inappropriate or suboptimal patient centring within the CT scanner gantry affects both image quality and radiation dose [22-26]. To our knowledge, however, there are no publications investigating the CT number variation as a function of the combined influence of vertical off-centring at different tube voltages and phantom sizes. This paper will compare these parameters across two CT scanners.

\section{Materials And Methods}

A combined head and body system performance phantom supplied with the GEMINI GXL system and a CIRS Model 062 were used in this study. The head phantom construction consisted of a $20 \mathrm{~cm}$ diameter polyvinyl chloride (PVC) cylinder filled with water with three sections: a multi-pin layer, physics and water layer. For the purpose of this study, only the water layer from the head phantom was scanned to enable direct comparison. The CIRS Model 062 phantom is made of water-equivalent material and consists of two nested disks (outer and inner) measuring $18 \times 18 \times 5 \mathrm{~cm}$ simulating a head and $33 \times 27 \times 5 \mathrm{~cm}$ (average of $30 \mathrm{~cm}$ in diameter) simulating an abdomen. In order to simulate a water phantom, all inserts were replaced with water balloons. This eliminated CT number interference from neighbouring inserts.

The combined head and body phantom was initially scanned at the iso-centre using a GEMINI GXL system, at $90 \mathrm{kVp}, 250 \mathrm{mAs}$ with $5 \mathrm{~mm}$ slice thickness. To mimic clinical imaging, a routine helical abdomen protocol was used with a large bowtie filter, 0.75-s gantry rotation time, 0.9 Pitch, $6 \times 1.5$ collimation, and $50 \mathrm{~cm}$ field of view (FOV). The table was raised 20, 40,60, and $80 \mathrm{~mm}$ above the gantry iso-centre. Scanning was repeated three times for each displacement to determine reproducibility by identifying spurious results. Imaging was then repeated at 120 and $140 \mathrm{kVp}$, using the same scanning protocol parameters and table movements due to scanner limitations (PET functionality), off-centring was only possible in the vertical direction above the gantry iso-centre, which is acknowledged as a limitation of the study. 
The CIRS Model 062 phantom was scanned using a Siemens Emotion 16-slice System, with the same experimental setup to enable comparison between the two scanners. Three kVp sets were predetermined by the manufacturer at 80,110 , and $130 \mathrm{kVp}$.

Uniformity testing is performed by evaluating the CT number for uniform water or water-equivalent phantom. COVID-19 restrictions and the geographical location of the scanners used in this study meant that the same phantom was unable to be scanned at both locations. However, the performance of the two phantoms should be the same as both are water phantoms. The phantom supplied with the GEMINI GXL system is a water-filled test object (or containing liquid water) and the CIRS phantom is constructed originally from a water equivalent material meeting the requirement of the test [27]. The CT number uniformity was examined at variable tube voltages and phantom sizes $(18,20$, and $30 \mathrm{~cm})$, using Image $\mathrm{J}$ software, to evaluate the impact of beam hardening artifacts [28]. Regions of interest (ROIs) in this test were positioned across the image in both $\mathrm{X}$ and $\mathrm{Y}$ axis and included one $\mathrm{ROI}$ that was placed in the centre of the phantom (Fig. 1). The peripheral ROls were placed $1 \mathrm{~cm}$ from the phantom edge to exclude any influence from the PVC housing of the phantom on CT number [29].

The differences in the CT number were calculated between corresponding anterior (upper) and posterior (lower) ROls in both axis to investigate any differences associated with phantom off-centring and ROls locations.

\section{Statistical Analysis}

The statistical paired $t$-test was used to compare the CT number change between the two different scanners and vertical off-centring positions (above the gantry iso-centre). The significance of the impact of phantom off-centring, and body size on the $\mathrm{CT}$ number was evaluated using analysis of covariance (ANCOVA). A $p$-value of $<0.05$ was considered statistically significant.

\section{Results}

\section{CT Number Uniformity}

The $t$-test demonstrated a significant difference between CT number uniformity performance for both scanners $(t=31.6, \mathrm{df}=10.96, p<0.001)$. The GEMINI GXL system demonstrated the greatest inconsistency. At the off-centring $80 \mathrm{~mm}$ above iso-centre, the CT number changed at the peripheral ROIs was up to $17.1 \mathrm{HU}$ cross the $\mathrm{X}$ axis at $90 \mathrm{kVp}$. From the gantry iso-centre, there was a symmetrical type change in the CT number across the phantom when ROIs had shifted away from the phantom centre; for example, the recorded HU were 16.1 and 16 for peripheral ROls in the $\mathrm{X}$ axis. However, the CT number change was 15.5, and 17.1 for the same peripheral ROls when the phantom was $80 \mathrm{~mm}$ vertically offcentred. A cross the $\mathrm{Y}$-axis, the greatest CT number change recorded for the peripheral ROls was $27.2 \mathrm{HU}$ at $80 \mathrm{~mm}$ vertical off-centring and $90 \mathrm{kVp}$. Furthermore, vertical off-centring had more influence on CT number symmetry across the $\mathrm{Y}$-axis compared to the $\mathrm{X}$-axis as the measured $\mathrm{CT}$ number change for 
peripheral ROls were 17.7 and $14.9 \mathrm{HU}$ (at $+Y$ and $-Y$-axis respectively), while 27.2 and $13 \mathrm{HU}$ were recorded at $80 \mathrm{~mm}$ off-centring for the same measured ROls.

The Siemens system performance was more consistent for both phantom sizes at the iso-centre. However, for the $30 \mathrm{~cm}$ phantom at $80 \mathrm{~mm}$ vertical off-centring and $80 \mathrm{kVp}$, a change in the CT number was found to be more prominent at peripheral ROls, reaching a maximum of $11.1 \mathrm{HU}$ at the X-axis and 14 $\mathrm{HU}$ in the $\mathrm{Y}$-axis. The symmetry of CT number change at peripheral ROls was not influenced by vertical off-centring across the $X$-axis ( $X$ and $-X$-axis), changing from 0.7 and $1.0 \mathrm{HU}$ at the gantry iso-centre to 11.1 and $11 \mathrm{HU}$ at $80 \mathrm{~mm}$ off-centring and $80 \mathrm{kVp}$. At the same time, a maximum 14 and $2.2 \mathrm{HU}$ change was recorded across the $+Y$ and $-Y$-axis at $80 \mathrm{~mm}$ vertical off-centring compared to 3.3 and $6.1 \mathrm{HU}$ at the gantry iso-centre, Fig. 2. This is indicative of CT number uniformity being influenced by two factors: vertical off-centring and phantom size.

\section{The combined effect of vertical off-centring and phantom size on CT number}

A plot of the CT number change at various vertical off-centring is shown in Fig. 3. Based on body size, the $30 \mathrm{~cm}$ CIRS phantom (combined discs) demonstrated the greatest CT number change when the phantom was vertically off-centred by $80 \mathrm{~mm}$ and imaged at lower tube voltage $(80 \mathrm{kVp})$. After adjustment for CT number change, the covariate, phantom size, was shown to be significantly related to CT number change with vertical off-centring $(F(2,39)=228.68, p<0.001)$. Adjusted R-squared explained that vertical offcentring and phantom size could account for $92 \%$ of the recorded variance and resultant CT number change between images.

In Fig. 4 (a), ROI A showed a decrease in the average CT number when the phantom shifted vertically and further away from the gantry iso-centre. At the same time, when ROI B was similarly off-centred, moving closer to the beam centre, a slight increase in the average CT number for the first two vertical increments steps was evidenced. The average CT number of the lower and the upper ROls increased by $4.8 \mathrm{HU}$ at 90 $\mathrm{kVp}, 4 \mathrm{HU}$ at $120 \mathrm{kVp}$, and 4.7 at $140 \mathrm{kVp}$ when the phantom was vertically off-centred to $80 \mathrm{~mm}$ (Fig. $4 b)$.

Two ROIs were located peripherally at the anterior and posterior abdominal aspect of the CIRS phantom (Fig. 5a). At $110 \mathrm{kVp}$ and with an $80 \mathrm{~mm}$ vertical shift, the mean difference in the CT number compared to the gantry iso-centre was $-5.3 \mathrm{HU}$ in the anterior ROI, and $4.3 \mathrm{HU}$ in the posterior ROI. Further, the absolute mean differences in the CT number between the anterior and posterior ROls was $10.7 \mathrm{HU}$ at 80 $\mathrm{kVp}$ and $6.8 \mathrm{HU}$ for 110 and $130 \mathrm{kVp}$ with an $80 \mathrm{~mm}$ vertical off-centring (Fig. 5b). The CT difference was observed to be greater at lower tube voltage and as the phantom was moved further from the iso-centre.

\section{Tube Voltage}

Unlike the GEMINI GXL system, data for the Siemens system showed that the tube voltage had no effect on the CT number for water at the gantry iso-centre. It was also observed that the CT numbers remained close for both higher voltage sets (110 and $130 \mathrm{kVp}$ ), whilst for the GEMINI GXL system, the CT number 
was closer for both 90 and $120 \mathrm{kVp}$ sets under-examined conditions (Fig. 2). Generally, the vertical offcentring in both systems had a more dramatic influence on CT number change at low tube voltage.

\section{Discussion}

\section{CT number uniformity}

There is no universally accepted value for CT number uniformity; however, the International Electrotechnical Commission (IEC) recommends the mean CT number of the central ROI should not deviate from the manufacturer's specified value by more than $\pm 4 \mathrm{HU}$ for each material measured [29] and that the difference in uniformity do not exceed more than $2 \mathrm{HU}$ from the baseline value [30].

The Siemens system demonstrated superior uniformity performance in comparison to the GEMINI GXL system, most likely due to the incorporation of beam hardening correction software. Typically, these corrections are automatically applied for various body sizes. However, the uniformity test results were not the same between units when the respective phantoms were off-centred from the gantry iso-centre. This indicates that beam hardening corrections are corrected and functional at the gantry iso-centre only.

\section{Vertical off-centring and phantom size}

The current study showed that the $20 \mathrm{~cm}$ water phantom had no more than a $0.5 \mathrm{HU}$ change in CT number with up to $60 \mathrm{~mm}$ of vertical off-centring when the ROI was located at the phantom centre. The results by Kalra et al. (2009) concur with these results [26]. Furthermore, a study by Sukupova et al. (2016) found that within $100 \mathrm{~mm}$ of vertical off-centring, there was no substantial change in the CT number; however, with $140 \mathrm{~mm}$ or more vertical off-centring, a $20 \mathrm{HU}$ change was recorded [31]. These findings can be explained whereby the amount of off-centring required to cause a CT number change for a ROI will depend on the alignment of phantom geometry and the bowtie filter size. For instance, the CT number for the $30 \mathrm{~cm}$ phantom demonstrated a change of $4.3 \mathrm{HU}$ at $80 \mathrm{~mm}$ off-centring and $80 \mathrm{kVp}$ compared to $1.4 \mathrm{HU}$ for the $18 \mathrm{~cm}$ phantom.

A study by Hsieh (2015) reported CT number differences for a $20 \mathrm{~cm}$ water phantom were 1, 1.8, and 2.7 $\mathrm{HU}$ at 40,60 , and $80 \mathrm{~mm}$ vertical off-centring, respectively [17]. Figure 4 (a) illustrates two ROIs A and B, which are subjected to beam hardening and the bowtie effect. Opposing effects were observed in the upper and lower half of the $20 \mathrm{~cm}$ phantom. As the ROI A was vertically off-centred away from the isocentre, the CT number decreased, while the CT number for ROI B increased as it becomes vertically offcentred towards the iso-centre. The difference in CT number between the two ROls demonstrated an increase in a factor of two. The difference at $80 \mathrm{~mm}$ of off-centring was calculated and divided by two, resulting in the value of $2.75 \mathrm{HU}$ at $120 \mathrm{kVp}$, which matches the result of Hsieh (2015) at the same distance [17]. This correlates to the principle function of the bowtie filter [32].

Two ROls were located at both peripheral edges, anteriorly and posteriorly, of the CIRS phantom as shown in Fig. 5 (a). These ROls demonstrated a larger change in the CT number as a function of vertical off- 
centring compared to the values obtained at the phantom centre. This highlights the significance of ROI location on CT number variability. Goodsitt et al. (2006) reported that the CT number is dependent on the ROI location and that the number can change by up to $11 \mathrm{HU}$ for a scanned simulated lung phantom [33]. Szczykutowicz et al. (2016) measured the CT number change in relation to body position and ROI location at a 40,60, and $100 \mathrm{~mm}$ off-centring above and below the gantry iso-centre [34]. They used two ROls (anterior and posterior) for each scanned area. In the abdominal scan, the mean difference in the CT number compared to the gantry iso-centre was $8 \mathrm{HU}$ obtained at the anterior ROI, and $-6 \mathrm{HU}$ at the posterior ROI with $60 \mathrm{~mm}$ off-centre.

In the current study, the mean difference in the CT numbers between the posterior ROI (2), and the anterior $\mathrm{ROI}(1)$ was $10.7 \mathrm{HU}$ with $80 \mathrm{~mm}$ off-centring at lower tube voltage ( $80 \mathrm{kVp})$. This differs from the study by Szczykutowicz et al. who reported a maximum of $19 \mathrm{HU}$ in the abdominal area, at $100 \mathrm{~mm}$ vertical offcentring above the iso-centre, asserting that the bowtie influence diminished gradually with vertical offcentring [34]. As a consequence, noise is known to be greater on the phantom side positioned furthest from the gantry iso-centre.

\section{Tube Voltage}

Using the GEMINI GXL system, the Ct number was demonstrated to be energy-dependent. In contrast, as the Siemens system applies an algorithmic correction, the CT number for water energy is independent for the average adult size. However, the CT number was shown to be energy dependent for body tissues other than water.

It emerges clearly that CT numbers are closer in value between 90 and $120 \mathrm{kVp}$ for the GEMINI GXL system. Whereas the Siemens system showed closer values for 110 and $130 \mathrm{kVp}$ when the phantom was off-centred from the gantry iso-centre. This finding reflects the degree of CT number deviation for different scanned body sizes with respect to standard adult size values. In other words, using the lower tube voltage for smaller patient size in the Philips system will result in less CT number deviation as a function of off-centring compared to standard adult values, while this deviation will be higher again using Siemens system and vice versa.

\section{Limitations}

The inability to use the same phantom to examine the performance of each scanner due to COVID-19 restrictions is noted as a limitation, however, the comparison is still applicable since both are water phantoms. The study examined two manufacturers only and further research to include a more comprehensive range of vendors is needed.

\section{Conclusion}

The Siemens system demonstrated acceptable CT number uniformity and energy independence for an adult-sized water phantom at the gantry iso-centre compared to the GEMINI GXL system. As expected, 
uniformity and energy dependence were influenced by vertical off-centring. The extent of the change in CT number as a function of vertical off-centring was shown to depend on the degree of the improper alignment of the bowtie filter. The influence was more prominent for the larger phantom size and at lower tube voltages. Furthermore, the CT number deviation was highly dependent on both the size and the location of the measured ROI, and this deviation was observed to be more evident in peripheral phantom areas. Radiologists and radiographers should remain aware of the potential of CT numbers variation associated with poor positioning, especially when there is dependence on CT number accuracy for tissue lesion characterisation. Further research is still needed to support or refute the results of this study.

\section{Declarations}

\section{Acknowledgement}

The authors would like to acknowledge staff from the Riverina Cancer Care Centre (RCCC) Wagga Wagga NSW, and the AFIA Radiotherapy and Nuclear Medicine Centre Amman Jordan, for their timely support. Special thanks to The Hashemite University.

- Funding: Not applicable

- Conflicts of interest/Competing interests: Not applicable

- Availability of data and material: Not applicable

- Code availability: Not applicable

- Ethics approval: Not applicable

- Consent to participate: Not applicable

Consent for publication: Not applicable

\section{References}

1. Subhawong, T.K., et al., Soft-tissue masses and masslike conditions: what does CT add to diagnosis and management? AJR. American Journal of Roentgenology, 2010. 194(6): p. 1559-1567.

2. Herth, F.J.F., et al., The Modern Art of Reading Computed Tomography Images of the Lungs: Quantitative CT. Respiration, 2018. 95(1): p. 8-17.

3. Zhang, R., et al., Quantitative accuracy of CT numbers: Theoretical analyses and experimental studies. Med Phys, 2018. 45(10): p. 4519-4528.

4. Meyer, M., et al., Virtual Unenhanced Images at Dual-Energy CT: Influence on Renal Lesion Characterization. Radiology, 2019. 291(2): p. 381-390.

5. Lev, M.H. and R.G. Gonzalez, 17 - CT Angiography and CT Perfusion Imaging, in Brain Mapping: The Methods (Second Edition), A.W. Toga and J.C. Mazziotta, Editors. 2002, Academic Press: San Diego. p. 
6. Samei, E. and N. Pelc, Computed Tomography Approaches, Applications, and Operations: Approaches, Applications, and Operations. 2020.

7. Levi, C., et al., The unreliability of CT numbers as absolute values. AJR Am J Roentgenol, 1982. 139(3): p. 443-7.

8. Hunter, T.B., G.D. Pond, and O. Medina, Dependence of substance CT number on scanning technique and position within scanner. Comput Radiol, 1983. 7(3): p. 199-203.

9. Zerhouni, E.A., et al., Factors influencing quantitative CT measurements of solitary pulmonary nodules. J Comput Assist Tomogr, 1982. 6(6): p. 1075-87.

10. Zheng, X., et al., Body size and tube voltage dependent corrections for Hounsfield Unit in medical Xray computed tomography: theory and experiments. Scientific Reports, 2020. 10(1): p. 15696. Retrieved from https://doi.org/10.1038/s41598-020-72707-y

11. He, T., et al., Computed Tomography Number Measurement Consistency Under Different Beam Hardening Conditions: Comparison Between Dual-Energy Spectral Computed Tomography and Conventional Computed Tomography Imaging in Phantom Experiment. Journal of computer assisted tomography, 2015. 39(6): p. 981-985. Retrieved from https://pubmed.ncbi.nlm.nih.gov/26196347 https://www.ncbi.nlm.nih.gov/pmc/articles/PMC4654266/

12. Boland, G.W., et al., Characterization of adrenal masses using unenhanced CT: an analysis of the CT literature. American Journal of Roentgenology, 1998. 171(1): p. 201-204. Retrieved from https://doi.org/10.2214/ajr.171.1.9648789

13. Park, J.J., B.K. Park, and C.K. Kim, Adrenal imaging for adenoma characterization: imaging features, diagnostic accuracies and differential diagnoses. Br J Radiol, 2016. 89(1062): p. 20151018.

14. Mohammed, A.R., et al., Dynamic perfusion CT parameters and delayed contrast washout CT in characterization of adrenal tumors: A comparative study. The Egyptian Journal of Radiology and Nuclear Medicine, 2017. 48(3): p. 707-716.

15. Hélénon, O., et al., How to characterise a solid renal mass: A new classification proposal for a simplified approach. Diagnostic and Interventional Imaging, 2012. 93(4): p. 232-245.

16. Khan, F.M., Khan's the physics of radiation therapy. Fifth edition. ed, ed. J.P. Gibbons, et al. 2014, Philadelphia, Pennsylvania: Wolters Kluwer.

17. Hsieh, J., Computed tomography : principles, design, artifacts, and recent advances. Third edition. ed. 2015, Bellingham, Washington: SPIE. 
18. Goodsitt, M.M., E.G. Christodoulou, and S.C. Larson, Accuracies of the synthesized monochromatic CT numbers and effective atomic numbers obtained with a rapid kVp switching dual energy CT scanner. Med Phys, 2011. 38(4): p. 2222-32.

19. Khan, F.M. and J.P. Gibbons, Khan's the physics of radiation therapy. 2014.

20. Kaasalainen, T., et al., Effect of patient centering on patient dose and image noise in chest CT. AJR Am J Roentgenol, 2014. 203(1): p. 123-30.

21. Radiation dose from multidetector CT. 2nd ed. ed. Medical radiology : diagnostic imaging, ed. D. Tack, M.K. Kalra, and P.A. Gevenois. 2012, Heidelberg ;: Springer.

22. Gudjonsdottir, J., et al., Efficient use of automatic exposure control systems in computed tomography requires correct patient positioning. Acta Radiol, 2009. 50(9): p. 1035-41.

23. Habibzadeh, M.A., et al., Impact of miscentering on patient dose and image noise in x-ray CT imaging: phantom and clinical studies. Phys Med, 2012. 28(3): p. 191-9.

24. Matsubara, K., et al., Misoperation of CT automatic tube current modulation systems with inappropriate patient centering: phantom studies. AJR Am J Roentgenol, 2009. 192(4): p. 862-5.

25. Funama, Y., et al., Image noise and radiation dose using an automatic tube current modulation technique at 64-detector computed tomography: effect of off-center patient position, bowtie filter type, and scan projection radiograph. J Comput Assist Tomogr, 2009. 33(6): p. 973-7.

26. Kalra, M.K., et al., In-plane shielding for CT: effect of off-centering, automatic exposure control and shield-to-surface distance. Korean journal of radiology, 2009. 10(2): p. 156-163.

27. Mansour, Z., et al., Quality control of CT image using American College of Radiology (ACR) phantom. The Egyptian Journal of Radiology and Nuclear Medicine, 2016. 47(4): p. 1665-1671. Retrieved from https://www.sciencedirect.com/science/article/pii/S0378603X16301619

28. Gulliksrud, K., C. Stokke, and A.C. Trægde Martinsen, How to measure CT image quality: Variations in CT-numbers, uniformity and low contrast resolution for a CT quality assurance phantom. Physica Medica, 2014. 30(4): p. 521-526.

29. International standard IEC, IEC 61223-3-5 Evaluation and routine testing in medical imaging departments, in part 3- 5: acceptance tests - imaging performance of computed tomography X-ray equipment. 2013.

30. International standard IEC, IEC 61223-2-6. Evaluation and routine testing in medical imaging departments, in part 2-6: acceptance tests - imaging performance of computed tomography X-ray equipment. 2006. 
31. Sukupova, L., D. Vedlich, and F. Jiru, Consequences of the Patient's Mis-centering on the Radiation Dose and Image Quality in CT Imaging - Phantom and Clinical Study. Universal Journal of Medical Science, 2016. 4: p. 102-107.

32. Liu, F., et al., Dynamic bowtie filter for cone-beam/multi-slice CT. PloS one, 2014. 9(7): p. e103054e103054.

33. Goodsitt, M.M., et al., Accuracy of the CT numbers of simulated lung nodules imaged with multidetector CT scanners. Medical physics, 2006. 33(8): p. 3006-3017.

34. Szczykutowicz, T.P., A. DuPlissis, and P.J. Pickhardt, Variation in CT Number and Image Noise Uniformity According to Patient Positioning in MDCT. AJR Am J Roentgenol, 2017. 208(5): p. 1064-1072.

\section{Figures}
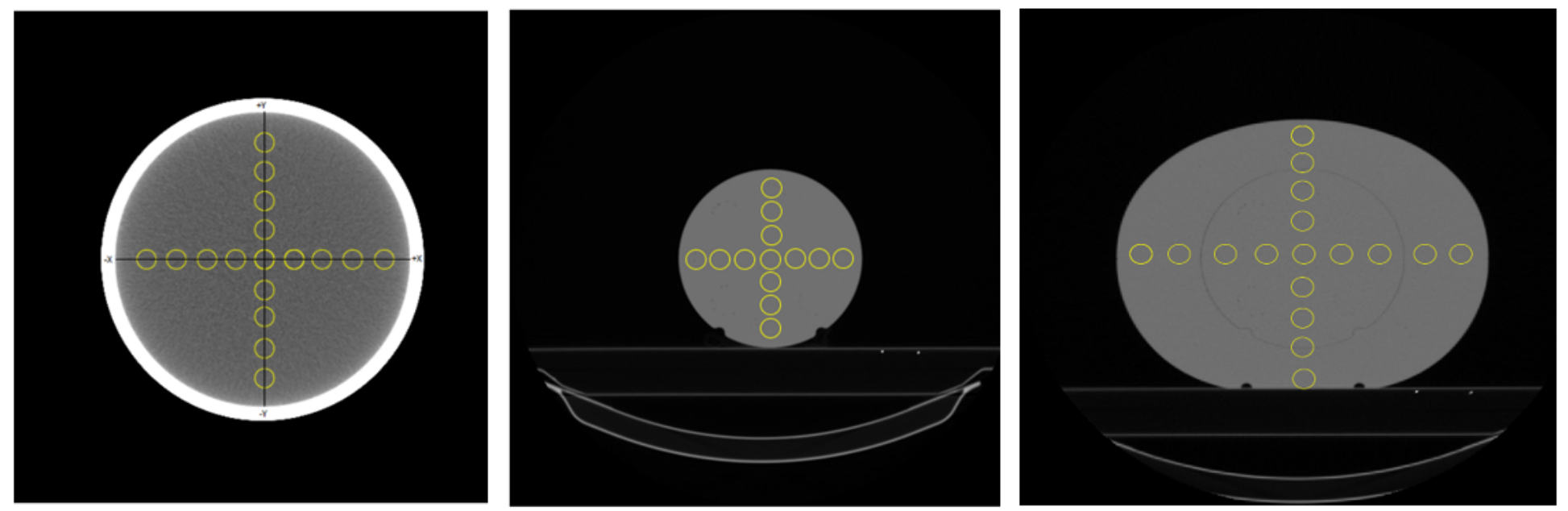

\section{Figure 1}

Placement of ROls across image filed ( $X$ and $Y$ axis) for uniformity measurements 

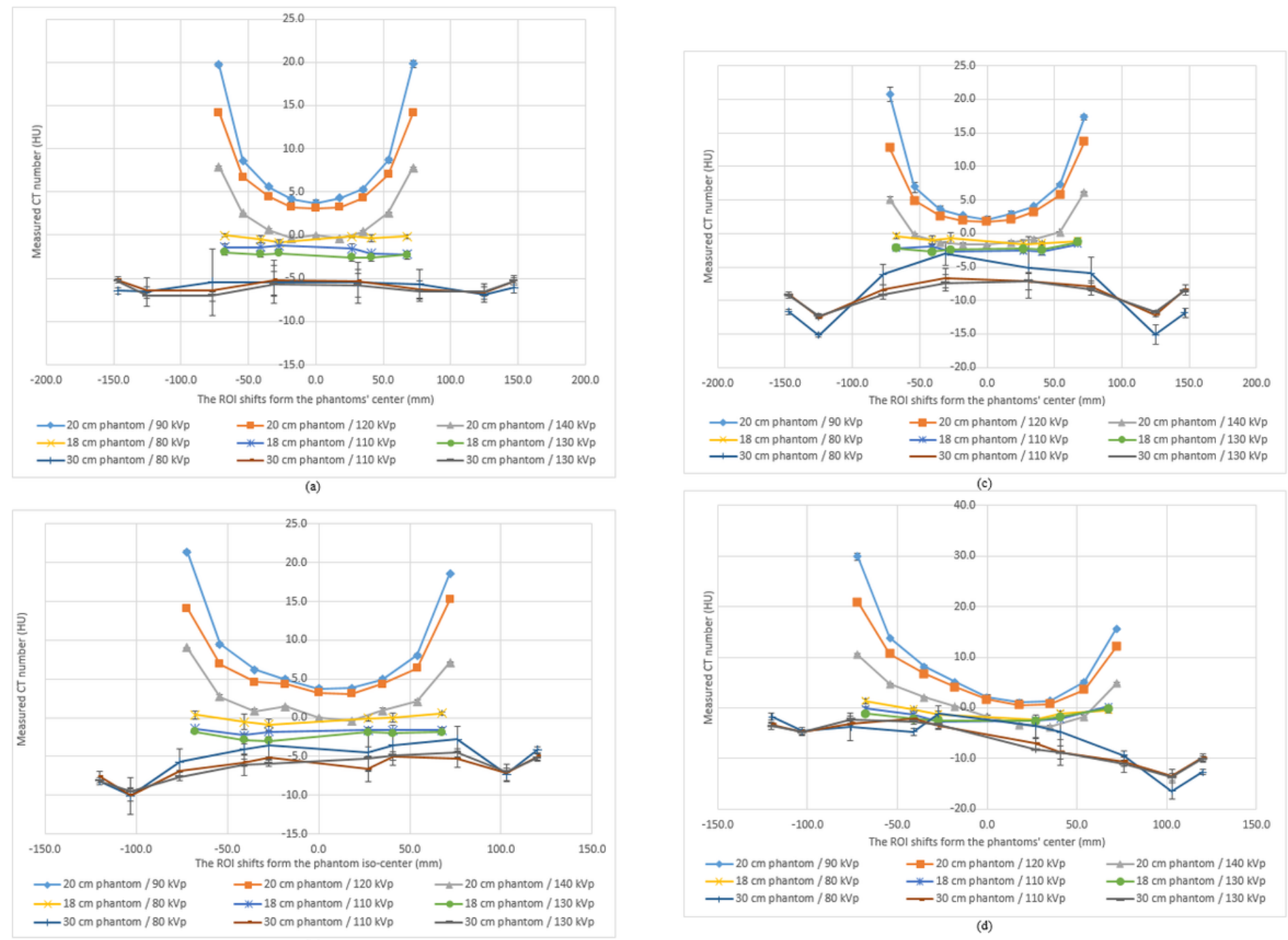

(b)

\section{Figure 2}

CT number uniformity across $X$ axis at (a) gantry iso-centre and (b) $80 \mathrm{~mm}$ off-gantry iso-centre. CT number uniformity across $Y$ axis at (c) gantry iso-centre and (d) $80 \mathrm{~mm}$ off-gantry iso-centre for both scanning systems using different tube voltages 


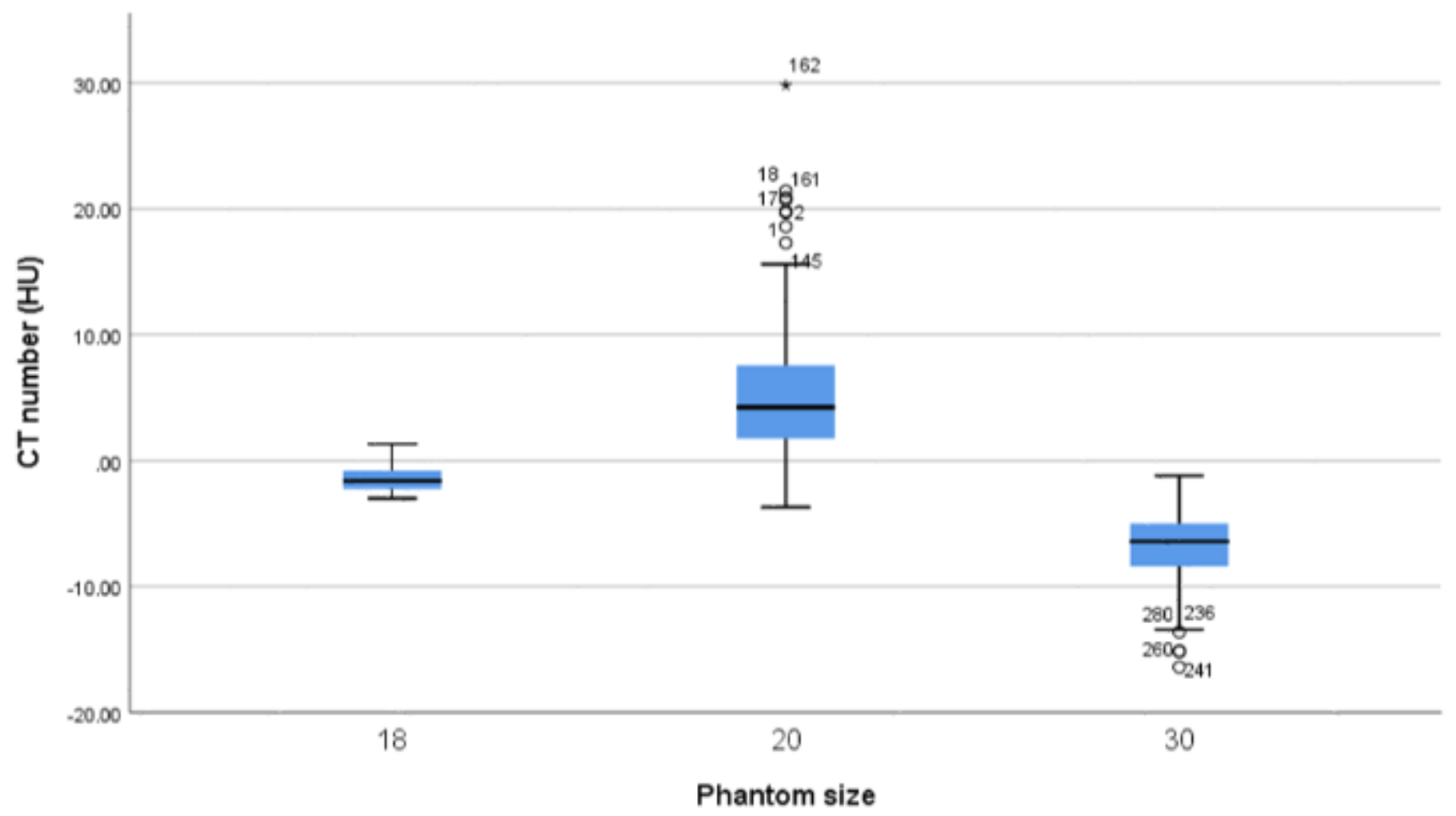

Figure 3

CT number change as a function of the vertical table off-centring for the three phantom sizes

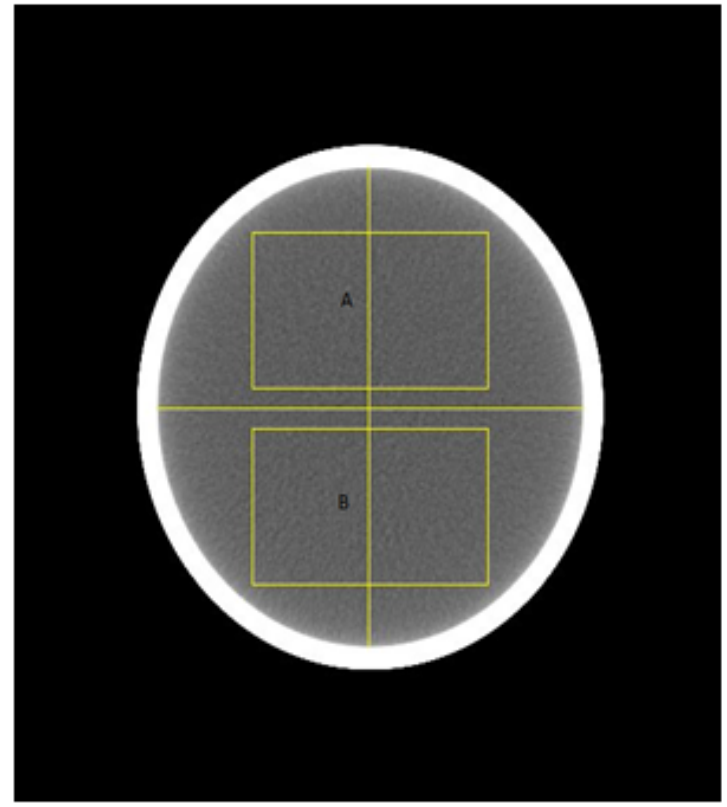

(a)

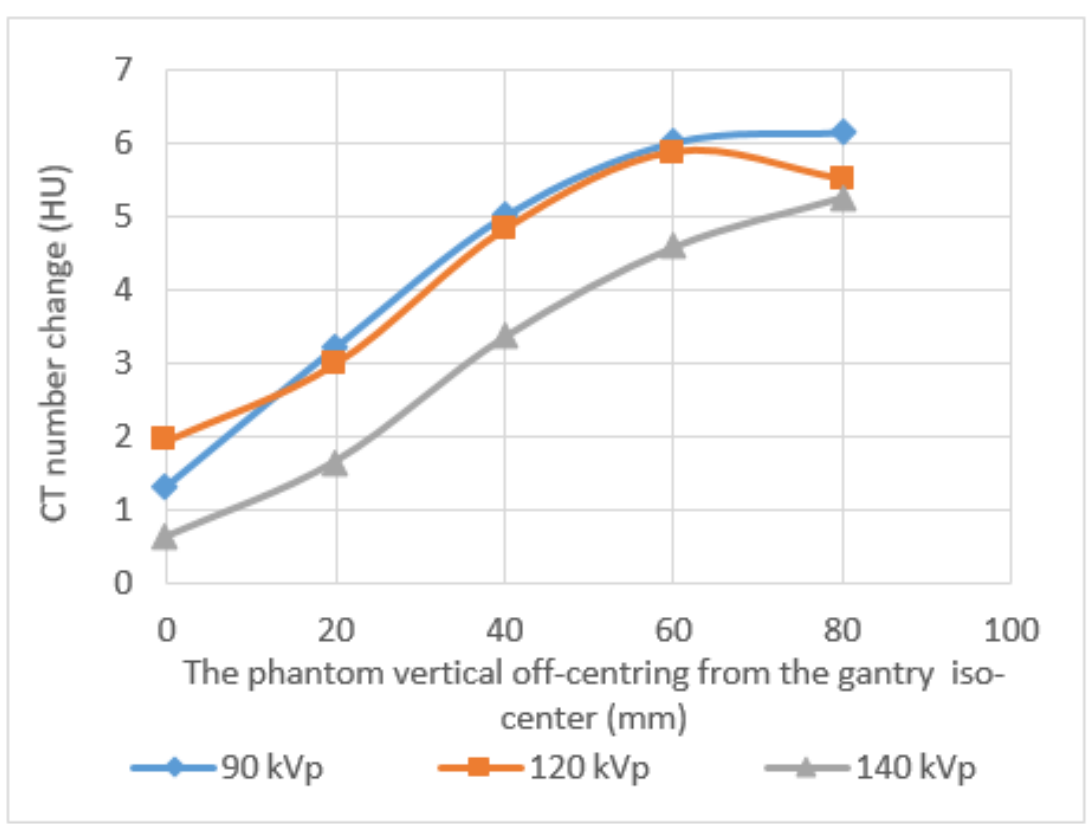

(b)

Figure 4 
(a) Two ROls defined on the $20 \mathrm{~cm}$ water phantom. (b) Graph showing differences between the mean CT number of the lower and the upper ROls as a function of phantom off-centring using the GEMINI GXL system

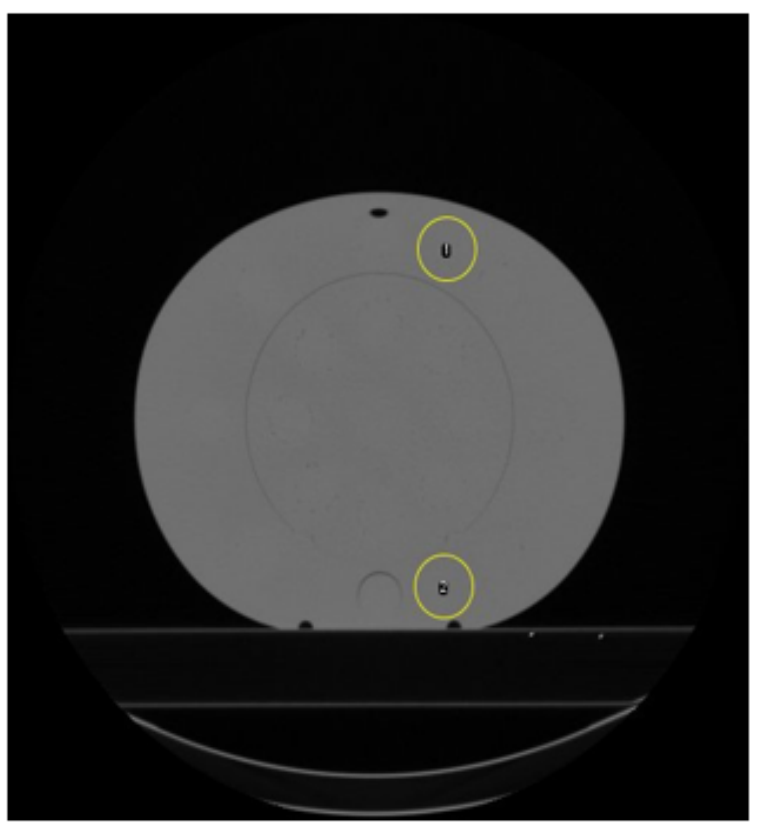

(a)

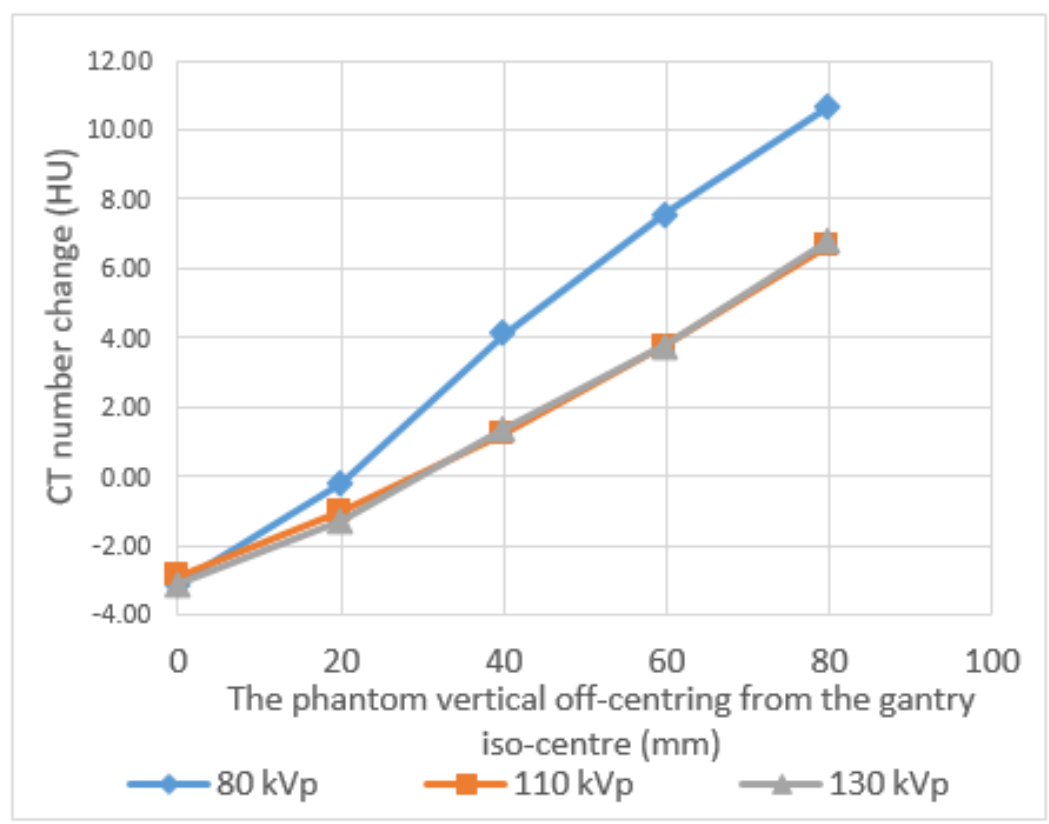

(b)

Figure 5

(a) Two ROls defined on a CIRS water phantom CT image used for calculating CT number difference. (b) The graph shows differences between mean of the CT number of the upper and the lower ROIs as a function of phantom off-centring using Siemens system 